\title{
Sub-barrier fusion cross section measurements with STELLA
}

M. Heine ${ }^{1, \star}, S$. Courtin $^{1,2}$, G. Fruet ${ }^{1}$, D.G. Jenkins ${ }^{3}$, D. Montanari ${ }^{1,2}$, P. Adsley ${ }^{3,4}$, C. Beck ${ }^{1}, S$. Della Negra ${ }^{4}$, P. Dené ${ }^{1}$, F. Haas ${ }^{1}$, F. Hammache ${ }^{4}$, G. Heitz ${ }^{1}$, O.S. Kirsebom ${ }^{5}$, M. Krauth ${ }^{1}$, J.

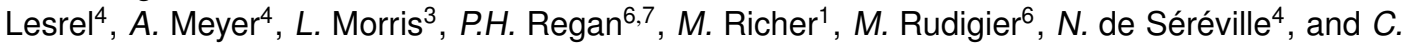
Stodel $^{8}$

${ }^{1}$ IPHC-CNRS/Université de Strasbourg, 67037 Strasbourg, (France)

${ }^{2}$ USIAS/Université de Strasbourg, 67083 Strasbourg, (France)

${ }^{3}$ University of York, YO10 5DD York, (UK)

${ }^{4}$ IPN d'Orsay, UMR8608, CNRS/IN2P3, PSUD 11, F-91406 Orsay, (France)

${ }^{5}$ Åarhus University, DK-8000 Åarhus, (Denmark)

${ }^{6}$ University of Surrey, GU2 7XH Guildford, (UK)

${ }^{7}$ National Physical Laboratory, Teddington, TW11 OLD, (UK)

${ }^{8}$ GANIL, CEA/DSM-CNRS/IN2P3, 14076 Caen, (France)

\begin{abstract}
The experimental setup STELLA (STELlar LAboratory) is designed for the measurement of deep sub-barrier light heavy ion fusion cross sections. For background suppression the $\gamma$-particle coincidence technique is used. In this project, $\mathrm{LaBr}_{3}$ detectors from the UK FATIMA (FAst TIMing Array) collaboration are combined with annular silicon strip detectors customized at IPHC-CNRS, Strasbourg, and the setup is located at Andromède, IPN, Orsay. The commissioning of the experimental approach as well as a sub-barrier ${ }^{12} \mathrm{C}+{ }^{12} \mathrm{C} \rightarrow{ }^{24} \mathrm{Mg}^{*}$ cross section measurement campaign are carried out.
\end{abstract}

\section{Introduction}

With the measurement of deep sub-barrier light heavy-ion fusion cross sections, both insights into nuclear cluster effects [1] as well as the $S$-factor at energies of astrophysical interest can be obtained. On the production route towards heavier elements during the carbon burning phase in massive stars, ${ }^{12} \mathrm{C}\left({ }^{12} \mathrm{C},{ }^{24} \mathrm{Mg}\right)$ was identified as a key reaction [2]. The relative energies of the fusion partners in the regime of astrophysical interest are as low as $E_{r e l}=2 \mathrm{MeV}$. This is far below the Coulomb barrier of ${ }^{12} \mathrm{C}$ at $E_{\text {rel }}=6.6 \mathrm{MeV}$ and the fusion cross sections for such measurements are expected to be in the order of nanobarn. Alongside to the use of high intensity beams up to $10 \mu \mathrm{A}$ and compacted detector configurations ${ }^{12} \mathrm{C}$ fusion is detected via the coincident measurement of evaporation products and de-excitation gammas [3] from daughter nuclei of the ${ }^{24} \mathrm{Mg}$ compound.

In the ${ }^{12} \mathrm{C}+{ }^{12} \mathrm{C}$ system the reaction channels, that include light charged particles, are

$$
\begin{aligned}
{ }^{12} \mathrm{C}+{ }^{12} \mathrm{C} \rightarrow{ }^{24} \mathrm{Mg}^{*} & \rightarrow{ }^{23} \mathrm{Na}+\mathrm{p}, \\
{ }^{24} \mathrm{Mg}^{*} & \rightarrow{ }^{20} \mathrm{Ne}+\alpha,
\end{aligned}
$$

^e-mail: marcel.heine@iphc.cnrs.fr 
with $Q$-values $Q_{\mathrm{p}}=2.24 \mathrm{MeV}$ and $Q_{\alpha}=4.62 \mathrm{MeV}$, respectively. Both reactions occur, in this energy domain, via the formation of an excited compound ${ }^{24} \mathrm{Mg}$ nucleus. It was shown that the de-excitation of (1) and (2) predominantly cascades through the first excited states of the daughter nuclei [4]. Alongside with the measurement of the corresponding particle energies the detection of the characteristic gamma lines at $440 \mathrm{keV}$ and $1634 \mathrm{keV}$, respectively, is targeted in the first instance.

\section{Measurement Station}

In the first campaign at Andromède [5], a ${ }^{12} \mathrm{C}^{2,3+}$ beam with energies ranging from $11.1 \mathrm{MeV}$ to $5.5 \mathrm{MeV}$ is directed on (thin) fixed targets of natural ${ }^{12} \mathrm{C}$ with densities of a few tens of $\mu \mathrm{g} / \mathrm{cm}^{2}$. In the course of the measurements the beam intensity was gradually increased from $40 \mathrm{pnA}$ to $800 \mathrm{pnA}$. The accuracy of the detection systems as well as the time alignment of the data acquisition are guaranteed and cross section measurements are carried out [6].

For the detection of de-excitation gammas, an array of 36 (1.5" x 2") $\mathrm{LaBr}_{3}$ detectors from the UK FATIMA collaboration is developed with the focus on the maximization of the detection efficiency. By means of Geant 4 simulations the geometrical acceptance was optimized to $21 \%$ of the $4 \Pi$ solid angle with the full-energy peak detection efficiency of $8 \%(440 \mathrm{keV})$ and $2 \%(1634 \mathrm{keV})$ [7]. The simulations are furthermore utilized to reconstruct the decay pattern of the natural contaminant ${ }^{138} \mathrm{La}$. In the comparison to experimental data a correction of an electronic walk of $10 \mathrm{keV}$ per day is carried out. This ensures that the QDC channels of the targeted gamma energies don't drift and a similar correction is foreseen for the charged particle detectors.

The nominal positions for annular in-beam double sided silicon strip detectors are $3 \mathrm{~cm}$ upstream as well as $6 \mathrm{~cm}$ up- and downstream with respect to the target are equipped with annular detectors based on S1, S3 Micron $®$ chips. They have a strip pitch of $1505 \mu \mathrm{m}$ (S1) and $886 \mu \mathrm{m}$ (S3) and are protected by absorber foils against scattered beam as well as electrons produced in the target. Due to the compact placement the geometrical acceptance is $25 \%$ of the $4 \Pi$ solid angle.

To withstand the heat input during bombardment with up to $10 \mu \mathrm{A}$ beam, a rotating target mechanism is developed and commissioned. Amongst fixed target positions the target wheel hosts three slots for frames with a diameter of $4.6 \mathrm{~cm}$ that spin with around $1000 \mathrm{rpm}$.

For the absolute normalization, two monitor detectors are placed in extensions around $25 \mathrm{~cm}$ from the beam spot position. These barrier surface silicon detectors measure the well know Mott scattering rates from the ${ }^{12} \mathrm{C}+{ }^{12} \mathrm{C}$ reaction at $45^{\circ}$. In addition, the beam current is recorded by an integrator behind the experiment. From a combination of both measurements, the target thickness can be recognized as well directly from the experimental data.

\section{First Results}

During the commissioning, the ${ }^{12} \mathrm{C}+{ }^{12} \mathrm{C}$ reaction is measured to compare with sub-barrier data from the literature and to guarantee the accuracy of the analysis. The time synchronization of the time stamped trigger less data acquisition system is tested extracting time correlations between gammas (Compton events) as well as the gamma- and particle detectors (fusion reactions). In this first attempt the time coincidence window is set to $400 \mathrm{~ns}$.

In the course of the experiment several calibration measurements with ${ }^{152} \mathrm{Eu}$ and ${ }^{241} \mathrm{Am}$ sources are carried out to guarantee the reliability of the energy gates for the gamma- and particle detectors, respectively. It turns out that a crude calibration at the beginning is sufficient for the gammas, while afterward data are calibrated in blocks of one hour based on the comparison to the simulated self activity pattern mentioned earlier. 
The correlation of simultaneous gamma- and charged particle detector entries is shown in figure 1 for a beam energy of around $E_{\text {beam }}=10.4 \mathrm{MeV}$ for a subset of data. The coincidence window of

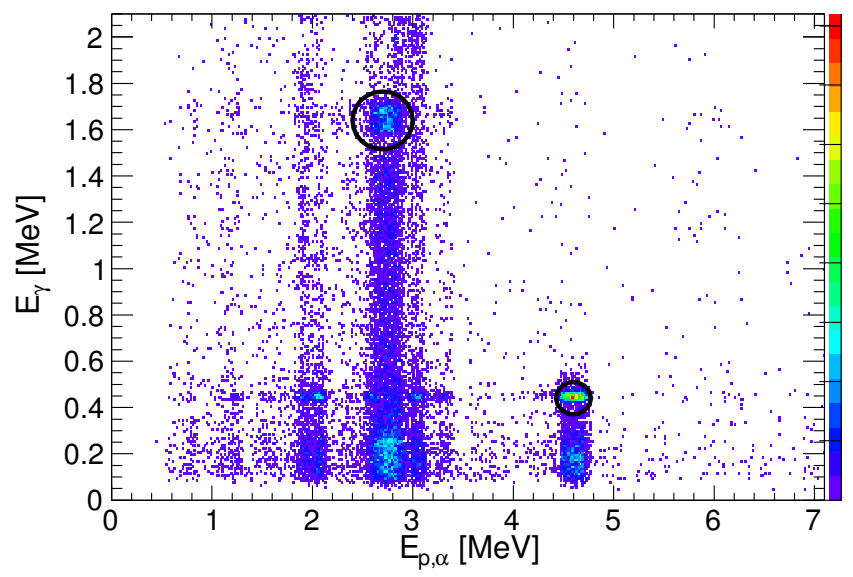

Figure 1. [Color online] Correlation of simultaneous gamma- and charged particle detector entries with a coincidence window of $400 \mathrm{~ns}$. Fusion events from the ${ }^{12} \mathrm{C}+{ }^{12} \mathrm{C}$ reaction, populating the first excited state of the ${ }^{23} \mathrm{Na}$ or the ${ }^{20} \mathrm{Ne}$ residual nucleus, can be selected like indicated for the characteristic gammas at $E_{\gamma}=0.440 \mathrm{MeV}$ or $E_{\gamma}=1.634 \mathrm{MeV}$ resulting from their subsequent de-excitation.

$400 \mathrm{~ns}$ used in this first analysis can still be minimized to reduce random coincidence background. For the calculation of fusion cross sections, the regions of interest around the full-energy depositions of the characteristic gammas from the ${ }^{23} \mathrm{Na}$ as well as ${ }^{20} \mathrm{Ne}$ daughter nuclei are highlighted with ellipses at the gamma energies of $E_{\gamma}=0.440 \mathrm{MeV}$ or $E_{\gamma}=1.634 \mathrm{MeV}$, respectively.

It is also noteworthy to mention that the timing resolution of the detection system allows for a discrimination of protons and alpha particles based on the rise time of the electronic pulse. In this way, a background free identification of the respective reaction is realized and a comprehensive analysis for further measurements at different beam energy is on the way.

\section{References}

[1] D. Jenkins and S. Courtin, Jour. Phys. G 42, 034010 (2015)

[2] A. Chieffi et al., Astr. Phys. Jour. 502, 737 (1998)

[3] C.L. Jiang et al., Nucl. Instr. and Methods A 682, 12 (2012)

[4] K.A. Erb et al., Phys. Rev. C 22, 22 (1980)

[5] S. Della Negra, Inn. Rev. p. 38 (2016)

[6] G. Fruet et al., submitted to EPJ Web of Conferences (2017)

[7] M. Heine et al., J. Phys. Conf. Ser 763, 012005 (2016) 
